\title{
Population distribution of six PCR-amplified loci in Madeira Archipelago (Portugal)
}

\author{
Francisco Corte-Real $^{\mathrm{a}, *}$, Luís Souto ${ }^{\mathrm{a}}$, M. João Anjos ${ }^{\mathrm{a}}$, \\ Mónica Carvalho ${ }^{a}$, Duarte N. Vieira ${ }^{a}$, Angel Carracedo ${ }^{\mathrm{b}}$, \\ M. Conceição Vide ${ }^{a}$ \\ ${ }^{a}$ Institute of Legal Medicine, Faculty of Medicine, University of Coimbra, Largo da Sé Nova, \\ 3000 Coimbra, Portugal \\ ${ }^{\mathrm{b}}$ Institute of Legal Medicine, Faculty of Medicine, University of Santiago de Compostela, \\ E-15705 Santiago de Compostela, Spain
}

Received 27 August 1998; received in revised form 9 November 1998; accepted 10 November 1998

\begin{abstract}
Frequency data of the short tandem repeat (STR) loci HUMTH01, HUMVWA31/A, HUMF13A1, HUMFES/FPS, D12S391 and HUMFIBRA/FGA were determined in blood stains obtained from a population of unrelated individuals from the Madeira Archipelago. The observed genotype distribution showed no significant deviation from the Hardy-Weinberg equilibrium and there was no evidence for association of alleles among the six loci. Population data showed a combined discrimination power of 0.9999998 and a chance of exclusion of 0.99597 . The frequencies are similar to those of other compared caucasian populations but significant differences were found between the Madeira population and Japanese, Chinese, Greenland Eskimos and Quechua Amerindians. The six loci studied, together proved to be highly discriminating and valuable for forensic cases. (C) 1999 Elsevier Science Ireland Ltd. All rights reserved.
\end{abstract}

Keywords: Short tandem repeats; HUMTH01; HUMVWA31/A; HUMF13A1; HUMFES/FPS; D12S391; HUMFIBRA/FGA; Population genetics; Madeira

\section{Introduction}

The STR polymorphisms HUMTH01 [1,2], HUMVWA31/A [3,4], HUMF13A1 [5], HUMFES/FPS [6], D12S391 [7], HUMFIBRA/FGA [8] are increasingly used for

\footnotetext{
*Corresponding author. Tel.: +351-39-854230; fax: +351-39-820549.

0379-0738/99/\$ - see front matter (c) 1999 Elsevier Science Ireland Ltd. All rights reserved.

PII: S0379-0738(98)00202-3
} 
paternity testing and forensic identification. All of these systems consist of tetranucleotide repeat units with some variants.

Allele frequency data from the relevant populations must accompany the use of genetic markers.

The Madeira population originates mainly from south of Portugal, Morocco and Algeria, since the 15th century. Emigration from Madeira to other places has been significant and more than 750000 individuals live in South Africa and Venezuela, where population databases can be useful to forensic investigations.

\section{Materials and methods}

DNA extraction was carried out from air-dried blood stains on cotton fabric obtained from unrelated individuals from Madeira Archipelago using Chelex method [9].

Multiplex PCR amplification of the TH01, VWA, F13A1 and FES/FPS [10] and singleplex amplification of the D12S391 and FIBRA/FGA loci used, per sample, $5 \mathrm{ng}$ of DNA, $200 \mu \mathrm{m}$ of each nucleotide (Pharmacia), $5 \mu \mathrm{l}$ of $10 \times$ buffer (Perkin-Elmer), 1.25 $\mathrm{U}$ Amplitaq polymerase (Perkin-Elmer and Dynazyme-Finn Zymes Oy), $0.15 \mu \mathrm{M}$ of primers VWA/ 1 and VWA/2, $0.18 \mu \mathrm{M}$ of primers TH01/1 and TH01/2, $0.16 \mu \mathrm{M}$ of primers $\mathrm{F} 13 \mathrm{~A} 1 / 1$ and $\mathrm{F} 13 \mathrm{~A} 1 / 2,0.055 \mu \mathrm{M}$ of primers FES/ 1 and FES/2, $0.25 \mu \mathrm{M}$ of primers D12/1 and D12/2, $0.25 \mu \mathrm{M}$ of primers FGA/1 and FGA/2 (Oswell DNA Service).

The PCR cycling conditions were (PE 480, PE 9600 thermocyclers):

TH01, VWA, F13A1, FES/FPS: 28 cycles of $95^{\circ} \mathrm{C}, 1 \mathrm{~min} ; 54^{\circ} \mathrm{C}, 1 \mathrm{~min} ; 72^{\circ} \mathrm{C}, 1 \mathrm{~min}$.

D12S391, FIBRA/FGA: 30 cycles of $94^{\circ} \mathrm{C}, 45 \mathrm{~s} ; 60^{\circ} \mathrm{C}, 1 \mathrm{~min} ; 72^{\circ} \mathrm{C}, 1 \mathrm{~min}$.

The samples were heat denatured at $95^{\circ} \mathrm{C}$ for $4 \mathrm{~min}$ before being loaded and electrophoresis was carried out in a $6 \%$ polyacrylamide sequencing gel on an ABI 373-A DNA Sequencer using the internal standard Genescan ROX (6-carboxyrhodamin) 2500 (multiplex detection) and 350 (to the D12S391 and FIBRA/FGA systems), during $6 \mathrm{~h}$ at constant power $(30 \mathrm{~W}, 2500 \mathrm{~V}$ and $40 \mathrm{~mA})$. Fragment sizes were determined automatically using the Genescan Software and typed by comparison with sequenced allelic ladders (allelic designation made according to the recommendations of the DNA Commission of the International Society for Forensic Haemogenetics).

Hardy-Weinberg equilibrium was tested with the exact test proposed by Guo and Thompson [11]. An unbiased estimate of heterozygosity was computed according to Nei [12], discrimination power according to Jones [13] and chance of exclusion according to Ohno et al. [14]. To test linkage disequilibrium it was used an exact test proposed on the Genepop program [15] and comparison of population data was carried out using an exact test with the STRUC program [16].

\section{Results and discussion}

The gene frequencies at the HUMTH01, HUMVWA31/A, HUMF13A1, HUMFES/ FPS, D12S391 and HUMFIBRA/FGA systems and the evaluation of the Hardy- 
Table 1

Allelic frequencies at the HUMTH01 system $(n=137)$

\begin{tabular}{llll}
\hline Allele & Frequency & Allele & Frequency \\
\hline 6 & $0.2226 \pm 0.0251$ & 9 & $0.1642 \pm 0.0224$ \\
7 & $0.1533 \pm 0.0218$ & 9.3 & $0.3029 \pm 0.0278$ \\
8 & $0.1533 \pm 0.0218$ & 10 & $0.0037 \pm 0.0037$ \\
\hline
\end{tabular}

Exact test: $P=0.0691 \pm 0.0019$.

Table 2

Allelic frequencies at the HUMVWA31/A system $(n=137)$

\begin{tabular}{llll}
\hline Allele & Frequency & Allele & Frequency \\
\hline 14 & $0.1277 \pm 0.0202$ & 18 & $0.1423 \pm 0.0211$ \\
15 & $0.1131 \pm 0.0191$ & 19 & $0.0730 \pm 0.0157$ \\
16 & $0.2591 \pm 0.0265$ & 20 & $0.0110 \pm 0.0063$ \\
17 & $0.2737 \pm 0.0269$ & & \\
\hline
\end{tabular}

Exact test: $P=0.0343 \pm 0.0012$.

Weinberg equilibrium in the Madeira population are presented in Tables 1-6. There is agreement between the observed genotype values and those expected under HardyWeinberg equilibrium $(P>0.01$ in the six systems).

With the exception of HUMFES/FPS, all the systems showed heterozygosity values $>70 \%$ (Table 7), the highest value being observed in the D12S391 marker (87.32\%). The six loci showed a combined chance of exclusion (CE) of 0.99597 and a combined discrimination power (DP) of 0.9999998, the systems D12S391 and HUMFIBRA/FGA being the most informative.

Table 3

Allelic frequencies at the HUMF13A1 system $(n=137)$

\begin{tabular}{llll}
\hline Allele & Frequency & Allele & Frequency \\
\hline 3.2 & $0.0657 \pm 0.0150$ & 12 & $0.0037 \pm 0.0037$ \\
4 & $0.0402 \pm 0.0119$ & 13 & $0.0037 \pm 0.0037$ \\
5 & $0.1861 \pm 0.0235$ & 14 & $0.0037 \pm 0.0037$ \\
6 & $0.2409 \pm 0.0258$ & 16 & $0.0073 \pm 0.0051$ \\
7 & $0.4197 \pm 0.0298$ & 17 & $0.0073 \pm 0.0051$ \\
8 & $0.0219 \pm 0.0088$ & & \\
\hline
\end{tabular}

Exact test: $P=0.4539 \pm 0.0083$.

Table 4

Allelic frequencies at the HUMFES/FPS system $(n=140)$

\begin{tabular}{llll}
\hline Allele & Frequency & Allele & Frequency \\
\hline 8 & $0.0071 \pm 0.0050$ & 12 & $0.2679 \pm 0.0265$ \\
10 & $0.2893 \pm 0.0271$ & 13 & $0.0321 \pm 0.0105$ \\
11 & $0.4036 \pm 0.0293$ & & \\
\hline
\end{tabular}

Exact test: $P=0.9726 \pm 0.0010$. 
Table 5

Allelic frequencies at the D12S391 system $(n=142)$

\begin{tabular}{llll}
\hline Allele & Frequency & Allele & Frequency \\
\hline 15 & $0.0282 \pm 0.0098$ & 21 & $0.1127 \pm 0.0188$ \\
16 & $0.0141 \pm 0.0070$ & 22 & $0.0810 \pm 0.0162$ \\
17 & $0.1127 \pm 0.0188$ & 23 & $0.0810 \pm 0.0162$ \\
18 & $0.2289 \pm 0.0249$ & 24 & $0.0247 \pm 0.0092$ \\
19 & $0.1479 \pm 0.0210$ & 25 & $0.0141 \pm 0.0070$ \\
20 & $0.1549 \pm 0.0215$ & &
\end{tabular}

Exact test: $P=0.1811 \pm 0.0043$.

Table 6

Allelic frequencies at the HUMFIBRA/FGA system $(n=145)$

\begin{tabular}{llll}
\hline Allele & Frequency & Allele & Frequency \\
\hline 18 & $0.0103 \pm 0.0059$ & 23 & $0.1552 \pm 0.0213$ \\
19 & $0.0414 \pm 0.0117$ & 24 & $0.1276 \pm 0.0196$ \\
20 & $0.1379 \pm 0.0203$ & 25 & $0.1207 \pm 0.0191$ \\
21 & $0.2345 \pm 0.0249$ & 26 & $0.0241 \pm 0.0090$ \\
22 & $0.1310 \pm 0.0198$ & 27 & $0.0035 \pm 0.0035$ \\
22.2 & $0.0069 \pm 0.0049$ & 28 & $0.0069 \pm 0.0049$ \\
\hline
\end{tabular}

Exact test: $P=0.1996 \pm 0.0056$.

The pairwise comparisons between loci showed no linkage disequilibrium $(P>0.01)$. Only pairwise test between HUMTH01-HUMF13A1 $(P=0.0387 \pm 0.0044)$ and HUMTH01-HUMFES/FPS $(P=0.0329 \pm 0.0031)$ showed $P$ values $<0.05$.

Comparisons of genotype values showed no significant differences $(P>0.01)$ between population data from this study and data from other caucasoid populations (Table 8), but we found statistical differences between Madeira population and Japanese, Chinese, Greenland Eskimos and Quechua Amerindians.

Table 7

Statistical parameters of forensic interest for the STRs studied

\begin{tabular}{llll}
\hline Systems & $h \pm$ S.E. & DP & CE \\
\hline HUMTH01 & $0.7810 \pm 0.0353$ & 0.91063 & 0.57505 \\
HUMVWA31/A & $0.7883 \pm 0.0349$ & 0.92801 & 0.61333 \\
HUMF13A1 & $0.7664 \pm 0.0362$ & 0.87365 & 0.49983 \\
HUMFES/FPS & $0.6857 \pm 0.0392$ & 0.83379 & 0.40596 \\
D12S391 & $0.8732 \pm 0.0279$ & 0.95674 & 0.72299 \\
HUMFIBRA/FGA & $0.7862 \pm 0.0341$ & 0.95749 & 0.70201 \\
Combined & & 0.9999998 & 0.99597
\end{tabular}

h, heterozygosity; DP, discrimination power; CE, chance of exclusion. 
Table 8

Genotype values comparisons between Madeira and other populations: exact test $(P \pm$ S.E. $)$

\begin{tabular}{|c|c|c|c|c|c|c|}
\hline Population compared & TH01 & VWA & F13 & FES & D12 & FGA \\
\hline Galicia, Spain* [17] & $0.605 \pm 0.005$ & $0.114 \pm 0.003$ & $0.695 \pm 0.006$ & $0.229 \pm 0.005$ & - & - \\
\hline Galicia, Spain* [7] & - & - & - & - & $0.376 \pm 0.004$ & - \\
\hline Catalonia, Spain $\dagger[18]$ & - & - & - & - & $0.863 \pm 0.003$ & - \\
\hline Italy* [19] & - & $0.085 \pm 0.003$ & - & $0.990 \pm 0.001$ & - & - \\
\hline North Italy $\dagger[20]$ & - & - & - & - & - & $0.996 \pm 0.000$ \\
\hline Switzerland* [21] & - & $0.066 \pm 0.002$ & - & $0.942 \pm 0.002$ & - & - \\
\hline Basel, Switzerland† [22] & $0.632 \pm 0.004$ & - & - & - & - & - \\
\hline Netherlands* [23] & - & - & - & - & - & $0.316 \pm 0.005$ \\
\hline Germany $\dagger[24]$ & - & - & - & $0.867 \pm 0.003$ & - & - \\
\hline SW Germany* [25] & $0.158 \pm 0.003$ & $0.029 \pm 0.002$ & - & - & - & - \\
\hline Münster, Germany* [7] & - & - & - & - & $0.569 \pm 0.005$ & - \\
\hline Germany $\dagger[26]$ & - & - & - & - & - & $0.587 \pm 0.006$ \\
\hline Caucasian, Austria $\dagger$ [27] & $0.299 \pm 0.004$ & $0.433 \pm 0.005$ & - & - & - & - \\
\hline Western Austria† $\left.{ }^{2} 28\right]$ & - & - & $0.637 \pm 0.006$ & $0.598 \pm 0.006$ & - & - \\
\hline Vienna, Austria* [29] & - & - & - & - & $0.626 \pm 0.005$ & - \\
\hline Caucasian, Austria* [30] & - & - & - & - & - & $0.040 \pm 0.002$ \\
\hline Caucasian, Britain $\dagger[31]$ & $0.284 \pm 0.004$ & $0.063 \pm 0.003$ & - & - & - & - \\
\hline Denmark* [32] & $0.026 \pm 0.001$ & - & - & - & - & - \\
\hline North Poland $\dagger[33]$ & $0.618 \pm 0.004$ & $0.171 \pm 0.004$ & - & $0.718 \pm 0.004$ & - & - \\
\hline Zagreb, Croatia $\dagger[34]$ & $0.371 \pm 0.004$ & $0.363 \pm 0.004$ & - & - & - & - \\
\hline Hungary $\dagger[35]$ & $0.534 \pm 0.005$ & $0.167 \pm 0.004$ & - & $0.928 \pm 0.002$ & - & - \\
\hline Barany., Hungary $\dagger[36]$ & - & - & - & - & - & $0.253 \pm 0.004$ \\
\hline Turkey† [26] & - & - & - & - & - & $0.558 \pm 0.005$ \\
\hline Morocco† [26] & - & - & - & - & - & $0.162 \pm 0.004$ \\
\hline Japan* [37] & $0.000 \pm 0.000$ & - & - & - & - & - \\
\hline Central Japan $\dagger[38]$ & - & $0.002 \pm 0.000$ & $0.000 \pm 0.000$ & - & - & - \\
\hline Tokyo, Japan* [39] & - & - & - & $0.000 \pm 0.000$ & - & - \\
\hline Japan† [26] & - & - & - & - & - & $0.232 \pm 0.004$ \\
\hline South China* [40] & $0.000 \pm 0.000$ & $0.000 \pm 0.000$ & - & - & - & - \\
\hline $\begin{array}{l}\text { Quechua Amerindians, } \\
\text { Bolivia* [41] }\end{array}$ & $0.000 \pm 0.000$ & $0.000 \pm 0.000$ & - & - & - & - \\
\hline Greenland Eskimos* [32] & $0.000 \pm 0.000$ & - & - & - & - & - \\
\hline
\end{tabular}

Comparison with observed* or expected $\dagger$ genotype values.

\section{Acknowledgements}

The authors are grateful to Dr J. Pita da Silva for his assistance on the collection of samples. We would like to thank Professors M.V. Lareu and J.I. Muñoz-Barús for their valuable comments.

\section{References}

[1] A. Edwards, A. Civitello, H.A. Hammond, C.T. Caskey, DNA typing and genetic mapping with trimeric and tetrameric tandem repeats, Am. J. Hum. Genet. 49 (1991) 746-756. 
[2] M.H. Polymeropoulos, H. Xiao, D.S. Rath, C.R. Merril, Tetranucleotide repeat polymorphism at the human tyrosine hydroxylase gene (TH), Nucleic Acids Res. 19 (1991) 3753.

[3] H.K. Ploos van Amstel, R.H. Reitsma, Tetranucleotide repeat polymorphisms in the vWF gene, Nucleic Acids Res. 18 (1990) 4957.

[4] C.P. Kimpton, A. Walton, P. Gill, A further tetranucleotide repeat polymorphism in the vWF gene, Hum. Mol. Genet. 1 (1992) 287.

[5] M.H. Polymeropoulos, D.S. Rath, H. Xiao, C.R. Merril, Tetranucleotide repeat polymorphism at the human coagulation factor XIII A subunit gene (F13A1), Nucleic Acids Res. 19 (1991) 4306.

[6] M.H. Polymeropoulos, D.S. Rath, H. Xiao, C.R. Merril, Tetranucleotide repeat polymorphism at the human c-fes/fps proto-oncogene (FES), Nucleic Acids Res. 19 (1991) 4018.

[7] M.V. Lareu, C. Pestoni, M. Schürenkamp, S. Rand, B. Brinkmann, A. Carracedo, A highly variable STR at the D12S391 locus, Int. J. Legal Med. 109 (1996) 134-138.

[8] K.A. Mills, D. Even, J.C. Murray, Tetranucleotide repeat polymorphism at the human alpha fibrinogen locus (FGA), Hum. Mol. Genet. 1 (1992) 779.

[9] P.S. Walsh, D.A. Metzger, R. Higuchi, Chelex®100 as a medium for simple extraction of DNA for PCR-based typing from forensic material, Biotechniques 10 (1991) 506-513.

[10] C. Kimpton, P. Gill, A. Walton, A. Urquhart, E.S. Millican, M. Adams, Automated DNA profiling employing multiplex amplification of short tandem repeat loci, PCR Methods Appl. 3 (1993) 13-22.

[11] S.W. Guo, E.A. Thompson, Performing the exact test of Hardy-Weinberg proportion for multiple alleles, Biometrics 48 (1992) 361-372.

[12] M. Nei, Estimation of average heterozygosity and genetic distance from a small number of individuals, Genetics 89 (1978) 583-590.

[13] D.A. Jones, Blood samples: probability of discrimination, J. Forensic Sci. Soc. 12 (1972) 355-359.

[14] Y. Ohno, I.M. Sebetan, S. Akaishi, A simple method for calculating the probability of excluding paternity with any number of codominant alleles, Forensic Sci. Int. 19 (1982) 93-98.

[15] M. Raymond, F. Rousset, Genepop (version 1.2): population genetics software for exact tests and ecumenicism, J. Hered. 86 (1995) 248-249.

[16] M. Raymond, F. Rousset, An exact test for population differentiation, Evolution 49 (1995) 1280-1283.

[17] C. Pestoni, M.V. Lareu, M.S. Rodríguez, I. Muñoz, F. Barros, A. Carracedo, The use of the STRs HUMTH01, HUMVWA31/A, HUMF13A1, HUMFES/FPS, HUMLPL in forensic application: validation studies and population data for Galicia (NW Spain), Int. J. Legal Med. 107 (1995) 283-290.

[18] M. Gené, A. Carracedo, E. Huguet, A. Pérez-Pérez, P. Moreno, Population genetics of the D12S391, CSF1PO and TPOX loci in Catalonia (Northeast Spain), Int. J. Legal Med. 111 (1998) 52-54.

[19] M. Dobosz, M. Pescarmona, A. Moscetti, A. Caglià, E. D'Aloja, L. Grimaldi, Allele frequencies of VWA, FESFPS, FXIIIA1 and D21S11 in an Italian population sample, in: A. Carracedo, B. Brinkmann, W. Bär (Eds.), Advances in Forensic Haemogenetics 6, Springer, Berlin, 1996, pp. 526-527.

[20] F. Betti, B. Giacomazzo, F. Ghio, A. Piccini, North Italian population genetic data on the STR system HumFGA, Int. J. Legal Med. 110 (1997) 110-111.

[21] A. Kratzer, W. Bär, Swiss population data for the STR systems HUMVWA, HUMF13A1 and HUMFES, in: A. Carracedo, B. Brinkmann, W. Bär (Eds.), Advances in Forensic Haemogenetics 6, Springer, Berlin, 1996, pp. 563-565.

[22] M.N. Hochmeister, J.M. Jung, B. Budowle, U.V. Borer, R. Dirnhofer, Swiss population data on three tetrameric short tandem repeat loci-VWA, HUMTH01, and F13A1—derived using multiplex PCR and laser fluorescence detection, Int. J. Legal Med. 107 (1994) 34-36.

[23] A. Ovington, P. Daselaar, M. Sjerps, A. Kloosterman, A Dutch population study of the STR loci D21S11 and HUMFIBRA, Int. J. Legal Med. 110 (1997) 14-17.

[24] W. Huckenbeck, H.G. Scheil, S. West, K. Demir, J. Kanja, A. Kaiser, et al., German data on the PCR based loci HUMVWA31, HUMTH01, HUMFES/FPS, HUMF13B and D1S80, in: A. Carracedo, B. Brinkmann, W. Bär (Eds.), Advances in Forensic Haemogenetics 6, Springer, Berlin, 1996, pp. 549-551.

[25] K. Leim, S. Degenhartt, W. Reichert, R. Mattern, Studies on the HUMTH01 and HUMVWA polymorphisms in a south west German population, in: A. Carracedo, B. Brinkmann, W. Bär (Eds.), Advances in Forensic Haemogenetics 6, Springer, Berlin, 1996, pp. 566-567.

[26] B. Rolf, K. Waterkamp, J. Hühne, Allele frequency data for the FGA locus in eight populations, Int. J. Legal Med. 111 (1998) 55-56. 
[27] M. Klintschar, M. Kubat, A study of the short tandem repeat systems HUMVWA and HUMTH01 in an Austrian population sample, Int. J. Legal Med. 107 (1995) 329-330.

[28] E. Ambach, W. Parson, H. Niederstätter, B. Budowle, Multiplex PCR and automated fluorescence detection of four tetrameric STRs in a Western Austrian population, in: A. Carracedo, B. Brinkmann, W. Bär (Eds.), Advances in Forensic Haemogenetics 6, Springer, Berlin, 1996, pp. 483-485.

[29] B. Glock, E.M. Dauber, D.W.M. Schwartz, W.R. Mayr, Additional variability at the D12S391 STR locus in an Austrian population sample: sequencing data and allele distribution, Forensic Sci. Int. 90 (1997) 197-203.

[30] F. Neuhuber, M. Klintschar, M. Radacher, A collaborative genetic study on the STR system FGA in two Austrian population samples, Forensic Sci. Int. 91 (1998) 1-6.

[31] I.W. Evett, P.D. Gill, J.A. Lambert, N. Oldroyd, R. Frazier, S. Watson, Statistical analysis of data for three British ethnic groups from a new STR multiplex, Int. J. Legal Med. 110 (1997) 5-9.

[32] L.J. Nellemann, A. Moller, N. Morling, PCR typing of DNA fragments of the short tandem repeat (STR) system HUMTH01 in Danes and Greenland Eskimos, Forensic Sci. Int. 68 (1994) 45-51.

[33] R. Pawlowski, A. Maciejewska, R. Paszkowska, Frequencies for five short tandem repeat (STR) systems in a population from North Poland, Int. J. Legal Med. 110 (1997) 10-13.

[34] M. Kubat, P. Wiegand, B. Brinkmann, Population genetic study from the Zagreb area using 3 STR systems, Int. J. Legal Med. 107 (1995) 219-221.

[35] J. Woller, S. Füredi, Z. Pádár, Hungarian population data for 11 PCR-based polymorphisms, in: A. Carracedo, B. Brinkmann, W. Bär (Eds.), Advances in Forensic Haemogenetics 6, Springer, Berlin, 1996, pp. 647-649.

[36] Z. Kozma, A. Nagai, J. Woller, S. Füredi, J. Sétáló, I. Ohya, K. Nishi, Fluorescence based coamplification and automated detection of the STR loci HUMFIBRA and HUMD21S11 in a Hungarian Caucasian population sample, Int. J. Legal Med. 111 (1998) 103-104.

[37] M. Takahashi, Y. Kato, G. Miyakawa, A. Kurosu, S. Kamiyama, Allele detection and population study in Japanese using two STR loci (CYP19 and HUMTH01), Int. J. Legal Med. 108 (1996) 321-322.

[38] A. Nagai, S. Yamada, Y. Watanabe, Y. Bunai, I. Ohya, Japanese population data on six STR loci, in: A. Carracedo, B. Brinkmann, W. Bär (Eds.), Advances in Forensic Haemogenetics 6, Springer, Berlin, 1996, pp. $587-588$.

[39] S. Nakamura, T. Sawaguchi, A. Sawaguchi, Forensic application of STR polymorphic markers, in: A. Carracedo, B. Brinkmann, W. Bär (Eds.), Advances in Forensic Haemogenetics 6, Springer, Berlin, 1996, pp. $589-591$.

[40] Y. Hou, H. Walter, Genetics substructure at the STR loci HUMTH01 and HUMVWA in Han populations, China, in: A. Carracedo, B. Brinkmann, W. Bär (Eds.), Advances in Forensic Haemogenetics 6, Springer, Berlin, 1996, pp. 468-470.

[41] M. Gené, E. Huguet, P. Moreno, M. Fuentes, J. Corbella, J. Mezquita, Aymara and Quechua Amerindian populations characterized by HUMTH01 and HUMVWA STR polymorphisms, in: A. Carracedo, B. Brinkmann, W. Bär (Eds.), Advances in Forensic Haemogenetics 6, Springer, Berlin, 1996, pp. 537-539. 